\title{
Teaching football-players the techniques of dribbling and stroke using digital technologies in terms of interactive train- ing complex
}

\author{
Vladimir Afonshin ${ }^{1}$, Gerold Drandrov ${ }^{2 *}$, Vladimir Burtsev ${ }^{3}$, Mikhail Polevchikov $^{4}$ \\ ${ }^{1}$ Mari State Technical University, 424007, Ioshkar Ola, Russia \\ ${ }^{2}$ Chuvash State Pedagogical University, 428000, Cheboksary, Russia \\ ${ }^{3}$ Volga region State Academy of Physical Culture, Sport and Tourism, 420010, Kazan, Russia \\ ${ }^{4}$ Mari State University, 424000, Ioshkar Ola, Russia
}

\begin{abstract}
Russian football players are inferior to football players from the leading European clubs in the level of dribbling and stroke mastering techniques. This drawback overcoming demands scientific search for the ways of teaching process development, taking into consideration modern achievements in the sphere of digital technologies. The aim of the research work is to create the methodology of teaching young football players the technique of dribbling and stroke using digital technologies. They are realized on the basis of an interactive training complex (ITC). The work presents the description of created by us ITC. It provides the following: an active polymedia information environment creation, which defines the direction and speed of an athlete's movements with the ball and without a ball at a training ground; an athlete's functional state characteristics and the training activity parameters registration. We created the methodology of ITC use. It includes the amount of new training exercises and the ways of the training activity organization. We present the results of testing the methodology. They prove its practical effectiveness. It is conditioned by the opportunities in the methodology: realization of methodical techniques of repeated, adjacent, closed up, contrast and variative tasks; control over the process and results of the training tasks fulfillment; regulating the difficulty of the tasks; training tasks fulfillment organization in terms of competitive method.
\end{abstract}

\section{Introduction}

The level of sports achievements of Russia national team and native football clubs in football world is still not very high [1]. In modern football the first place is given to speed dribbling with the stroke of two-three opponents. The football players of the leading European clubs have a high level of these techniques mastering. It helps the team to solve successfully tactical problems of creating the situations of playing advantage creation close to the goal of the opponent and realize them while attacking the goal. The observed lagging behind among native football players in these components of the attacking game technique proves a low level of traditional approaches pedagogical effectiveness to teaching the technique of dribbling and stroke [2]. This difficulty overcoming is an urgent objective of coaches' practical activity improvement. They train sports reserve in football. On the other hand, it conditions the necessity to search for new, more effective technologies of teaching these techniques [3].

In terms of all spheres of life digitalization the reasonable direction of sports training system development becomes digital technologies creation and use. That is why the leading world sports states, realizing the increasing role of a qualitative training of sports reserve, improve their resources in this direction. Nowadays this country is inferior to its rivals in innovative sports technologies development and introduction. They use modern achievements in the sphere of informatization $[4,5]$.

We supposed that increasing the effectiveness of teaching football technique is possible owing to digital technologies use on the basis of interactive training complexes creation. They have multimedia equipment and software program. It broadens and changes the opportunities of motor actions teaching process informational support [6, 7]. Their use during educational-training process would help to model different variants of competitive situations spatiotemporal structure and teach technical-tactical techniques in conditions maximum close to game conditions.

Moreover, realized by us abstract review of the literature showed that in spite of the supposed by us high didactic potential of digital technologies use in terms of ITC in teaching motor actions, the questions of their creation and use are not studied enough. That is why our research work is directed toward ITC and the methodology of its use creation in teaching young football players the technique of dribbling and stroke.

\footnotetext{
*Corresponding author: gerold49@mail.ru
} 


\section{Materials and methods}

During the research we took into account the principle regulations of "conjugate influence" and "dynamic correspondence", the concept of "artificially managing environment"; the theory and methodology of teaching motor actions; scientific works in the sphere of training simulators use in teaching physical exercises, its information and program support, knowledge of theory and methodology of football.

ITC creation and organization provided the existing optical means of information reflection (OMIR) study, video control and analysis means (VCAM), and their adaptation to the objectives of teaching techniques of dribbling and stroke; the applied and software program creation for OMIR and VCAM interaction; creating the package of the applied programs of diagnostics, interactive training lessons testing and organization.

Theoretical modeling was used for creating the methodology of teaching the technique of dribbling and stroke using ITC.

The quality of mastering the ways of movement stop and turn), techniques of dribbling and stroke was studied using expert estimation method and video with computer analysis.

Together with qualitative estimation we organized pedagogical testing of speed, speed-power oriented and coordinating abilities indices. We used traditionally used testing exercises: 15 meters running; 30 meters running; shuttle running 3x10 meters; zigzag running; standing long-jump; standing triple jump. The quality of mastering the technique of footpace stop and the technique of a turn during running was estimated according to the difference between the results of 30 meters running, on the one hand, and shuttle running $3 \times 10 \mathrm{~m}$ and zigzag running, on the other hand. In order to estimate the indices of mastering "dribbling" technique we used the following test exercises: 30 meters dribbling, dribbling in shuttle running $3 \times 10 \mathrm{~m}$, dribbling in zigzag running. Together with these parameters we took into account the difference in time of dribbling in running straight with the time of dribbling in shuttle running and zigzag running. For measuring the indices of mastering "stroke" technique we used the following test exercises: the respondent had to go from one side of the ground to another (15 meters distance) using dribbling and stroke in terms of the opponent.

The pedagogical experiment was held during one academic year at a sports school for children and teen-agers for substantiating the effectiveness of created by us methodology. Two groups of football players (10-11 years-old), who studied at the initial training groups of a sports school for children and teen-agers, took part in the experiment. The control group (18 people) studied the technique of dribbling and stroke taking into account the program of sports training for children and teen-agers sports school $[8,9,10]$. The football players from the experimental group (16 people) studied the technique of dribbling and stroke on the basis of the methodology using interactive training complex. Training lessons in the groups were held three times a week (duration 75-105 minutes). The indices of motor abilities and the quality of mastering the techniques of dribbling and stroke were estimated among the football players from both groups before and after the experiment. The validity of differences between the average group values was calculated using Student t-test and Pearson criterion.

\section{Results and discussion}

We created ITC, which helps to create an interactive and polymedia external environment (IPEE). It controls the training space organization. And registers the indices of a football-player's functional state and the characteristics of his activity. The choice of technical equipment of IPEE creation was held taking into account their orientation, on the one hand, and the problem of the training space formation, on the other hand - the objectives of its polymedia content formation and control.

ITC structure includes three independent parts (picture 1). The first part provides the training process and includes the following: playing field 1, which restricts the space of training; projective system, which includes all playing ground and color figures generator 2 and projector 3, which are used for dynamically lit objects formation on field 1 (permitted -4 and forbidden- 4 *parts), which show a football player 5 his possible position for the tasks of a coach fulfillment; speaker phone channel, which includes speech correction block 6 and loudspeaker 7 for speech instructions transfer or musical support.

On field 1projection system provides clear, convenient for perception optical coding of playing space of the moving on the field parts illumination. It shows necessary movements of a football player in accordance with the program of training; at the same time, the parts can differ in form, size, and color. In particular, with the help of such illumination it is possible to create light routes or give spatial positions, in which a football player, who has the ball, should be during the definite moment of the training task fulfillment. For this purpose permitted zones 4 are formed on fiend 1, an athlete should move into these zones; and forbidden zones $4^{*}$, where an athlete can't move. At the same time forbidden zones can have opponents or obstacles, which an athlete should overcome. A coach (Co) has an opportunity to change the position, form and square of the permitted and forbidden zones randomly for a football player with the help of the generator 2. We can suppose many different situations at the training space. They help to diversify the range of training exercises.

The second part of the complex, which helps to control a football player's functional state and the parameters of his activity during the training, includes measuring converters of the chosen physiological parameters 8 and sensing elements 9 of spatio-temporal characteristics of the training tasks fulfillment determination. They are fixed on an athlete 
and the signals from them go into the processing unit 10. Taking into account the registered parameters of an athlete's state and activity we measure the parameters of the training tasks, set with the help of projective system and sound radiators.

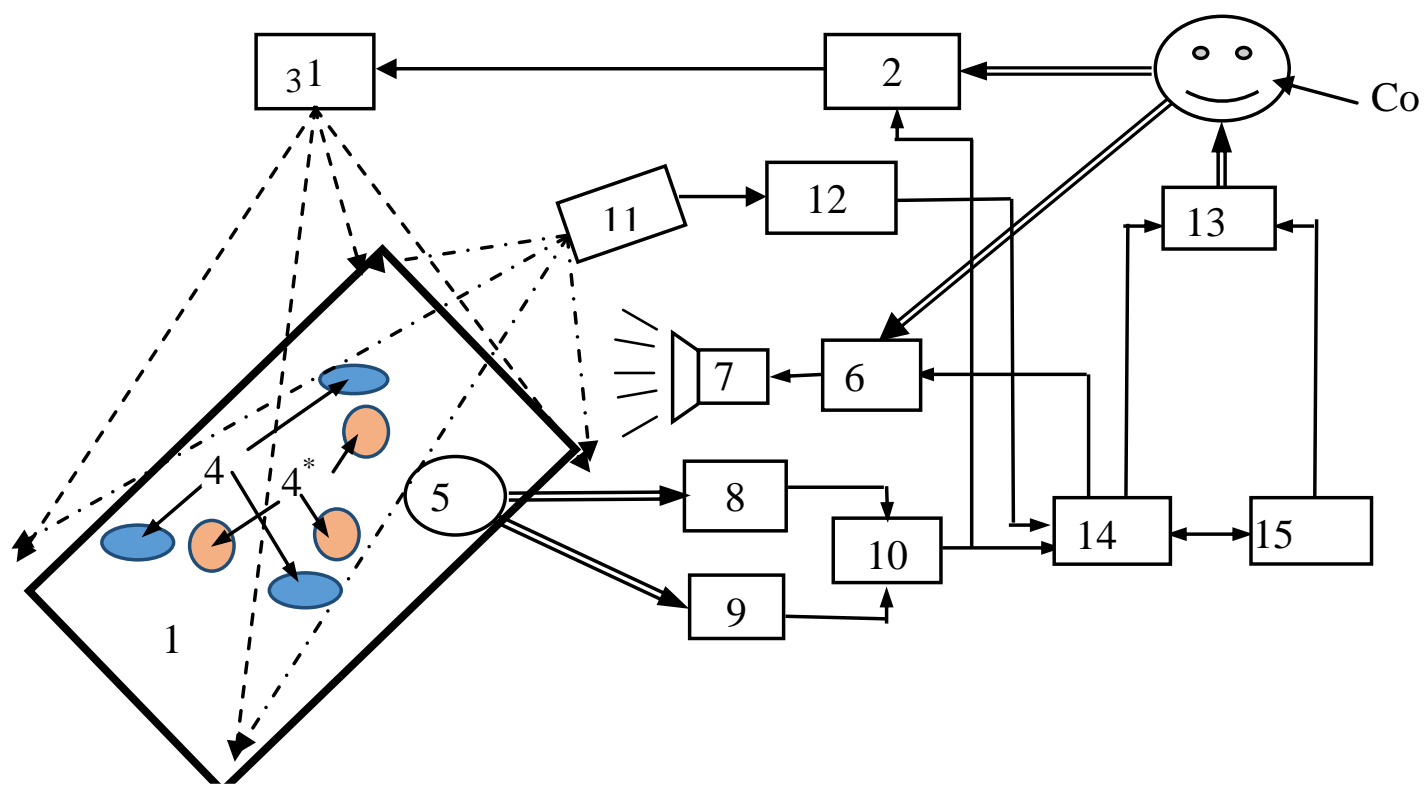

Fig. 1. Structure of an interactive training complex

For video information analysis, concerning the actions of an athlete, the third part of a complex is used. The third part includes several blocks. The whole process of training is registered by video camera 11, video signals from it after handling in gadget 12 (all sounds and unnecessary objects are taken from signals) go to computer 14 . In monitor 13 a coach sees the whole information concerning the training process, including the results about the athlete's state and activity. Simultaneously this information is recorded to external memory block 15 for further detailed analysis and a coach has an opportunity to ask for information from this block. While using the regimen with an active biological feedback, information from block 10 can directly go to generator 2 . Visual control with the help of video camera 11 helps a coach purposefully correct the positions of the permitted and forbidden zones and according to the ability of an athlete to be in a necessary place judge the quality of the tasks fulfillment.

Created by us ITC belongs to the class of biotechnical systems, as its structure includes biological objects - athletes; parameters of their behavior and state determine the program of training. It is protected by the range of the Russian Federation patents, awards were received at inventions and innovations exhibitions in several foreign countries [9, 10]. Successful testing of the training simulator was realized in some sports games and we received positive reviews from coaches and experts.

We created an innovative methodology of teaching young football players the technique of dribbling and stroke using ITC. ITC use helps to broaden the range of the training means (exercises) for a successful solution of the following pedagogical problems: 1) to teach the technique of movement without the ball; 2) to teach the techniques of dribbling and stroke.

As an example we would present the exercises for teaching the ways of movement:

Exercises at a "checkerboard pattern". With the help of dynamically lit illumination the field is divided into squares as the checkerboard pattern. Football players can move from square to square with the help of running, cross, chasse steps in different combination and different direction. The routes of movement are given by a coach or software complex with the help of illumination of those squares, which should be stepped on. Illumination moves from square to square, making the route with the speed equal to football player's maximal speed of movement.

Exercises with the "permitted zones". The aim of this group of training tasks is in constant being of a football player within the illuminating zone. The direction and the speed of movement of which changes both by a coach and with the help of software complex, taking into account motor abilities of an athlete. The exercise fulfillment demands maximum speed running from a football player and unexpected stops with the step and turns.

"Hillocks running". With the help of dynamic illumination "hillocks" are lit (light spots with the diameter till 0,5 m) on the field within a running step distance from the previous "hillock". An athlete has to move from one side of the field to another stepping on that "hillocks".

Exercise "hare chase". With the help of dynamic illumination "a hare" are placed on the field. It moves according to zigzag route. The speed of "hare" movement is almost equal to the speed of young football players running. "Hare" movement is reflected by dynamically lit illumination in a form of the radiant corridor (width from 0,5 till $1 \mathrm{~m}$ ). It follows the "hare" like a tail. A football player should catch the "hare", not leaving the bounds of the corridor. 
Exercises "ground overcoming with the forbidden zones". Light zones, both stationary and moving ones, are forbidden and model the position of the players on a football field. The aim of the training tasks is in maximal quick movements from one side of the field to another escaping the forbidden zones.

Game with "forbidden zones". Football players stand in pairs, one runs and the other football player should catch him and touch with the hand. Football players run on the field, escaping forbidden zones. If a person, who runs steps on the forbidden zone, he is considered caught and the players change their roles. If a person, who catches steps on the forbidden zone, a person, who runs has the right to step on the forbidden zone one time.

Exercise "ladder with side and forward escape after the feint". With the help of dynamic illumination three ladders are placed close to each other. The player is in the mid square. The opposite square is forbidden. The player fulfills slowly the step into the right side square. After that with some inertial pause forbidden becomes the opposite square of the side ladder. The player quickly returns into the mid square and steps forward, till the front square becomes forbidden. Then the same exercise is fulfilled into the opposite side.

ITC and dynamic illumination help to form the route of a football player with dribbling and set the distance, when the following kick doesn't lead to the running movements structure violation. It broadens the variety of the training exercises in order to teach dribbling and stroke.

Exercises with dribbling on "ladder". With the help of dynamic illumination a "ladder" is placed on the football field. The sizes of the squares are equal to the running step of a player. The players have to push the ball on the set by the squares of the ladder distance during the exercises with dribbling fulfillment.

Exercises with dribbling on "checkerboard pattern". With the help of dynamic illumination the squares on the field are marked as on a checkerboard pattern. The sizes of the squares are equal to the running step of the player. The routes of dribbling are set with the help of additional illumination of those squares, on which it is necessary to place the ball. Transfer from square to square is realized with one touch of the ball. Illumination moves from square to square giving the route with the speed. It is equal to maximal possible speed of football player movement with dribbling.

Dribbling "according to zones". With the help of dynamic illumination on the field zones are lit before the player at the distance of the running step from another zone. The player has to push the ball from one zone into another with the power. It would allow him fulfill the following touch of the ball during his entrance into this zone. The moment of a new zone appearance corresponds with the moment of ball touching during its push from the previous zone. The player has to move from one side of the field to another, moving with the ball from zone to zone.

Dribbling exercises with "hare chase". With the help of dynamic illumination on the field a "hare" is placed. It moves according to zigzag route. "Hare" movement is reflected in a form of the lit corridor (width 0,5 till $1 \mathrm{~m}$ ). It follows the "hare" like a tail. While dribbling an athlete has to push (stop, touch) the ball, when it is on a "hare".

On each line of a zigzag it is permitted to fulfill the definite amount of ball touch (from 1 till 3 ). The ball during dribbling shouldn't leave the corridor.

Dribbling exercises with "overcoming the field with the forbidden zones". Stationary and mobile light zones are forbidden and model the position of the players on a football field. The player has to move while dribbling using different ways, from one side of the playing ground to another, escaping the forbidden zones.

Exercises with sideways escape from the "forbidden zone". The forbidden zone moves towards the football player, who is moving forward dribbling. The football player fulfills a sideways step taking the ball and pushing it with one touch missing the forbidden zone, then continues moving dribbling, coming close to the next moving towards him forbidden zone.

The exercise of dribbling and stroke "ladder with side escape and forward after the feint". With the help of dynamic illumination three ladders are placed close to each other on the field. The player with the ball is in the mid square. The opposite square is forbidden. The player slowly fulfills the step to the right square pushing the ball into it. After that with some inertial pause forbidden becomes the opposite square of the side ladder. The player quickly returns with the ball to the mid square, and pushes the ball forward till the forward square becomes forbidden. Then the same exercise is fulfilled on the opposite side.

The results of the pedagogical experiment show high pedagogical effectiveness of the created by us methodology of teaching the technique of dribbling and stroke using ITC.

Table 1presents motor abilities indices of football players after the pedagogical experiment. It was stated that football players from the experimental group have some advantage in running results at 15 and 30 meters distance (for 0,07 and 0,05 seconds).

In shuttle running $3 \times 10 \mathrm{~m}$ and zigzag running the advantage of football players from the experimental group is valid and is 0,20 and 0,23 seconds. The level of speed-power oriented and power abilities development among football players from both groups didn't differ much. Difference indices between the results of straight 30 meters running, on the one hand, shuttle running 3x10 meters and zigzag running, on the other hand, is significantly lower among football players from the EG - 4,00 and 2,75 seconds against 4,15 and 2,93 seconds among football players from the CG.

Analyzing these results we come to the conclusion that successful mastering the ways of turns fulfillment and stops through exercises by football players from the EG in the training space, created with the help of ITC, was demonstrated in the results of testing exercises fulfillment. It is possible that high results of football players in shuttle running and zigzag running are also conditioned by other factors - the process of coordinating abilities development intensification.

Table 1. The indices of motor abilities in the respondents after the pedagogical experiment, $\mathrm{X} \pm \delta$

\begin{tabular}{l|l} 
Testing exercises & Groups of respondents \\
\hline
\end{tabular}




\begin{tabular}{|l|c|c|}
\hline & CG, $\mathrm{n}=18$ & EG, $\mathrm{n}=16$ \\
\hline 15 meters running, seconds & $3,47 \pm 0,10$ & $3,40 \pm 0,11$ \\
\hline 30 meters running, seconds & $5,70 \pm 0,22$ & $>0,05$ \\
\hline Shuttle running 3x10 meters, seconds & $9,85 \pm 0,20$ & $5,65 \pm 0,17$ \\
\hline Zigzag running, seconds & $8,63 \pm 0,21$ & $9,65 \pm 0,25$ \\
\hline Standing long-jump, cm & $166,1 \pm 7,2$ & $8,40 \pm 0,25$ \\
\hline Standing triple jump, cm & $491,0 \pm 12,1$ & $169,7 \pm 8,3$ \\
\hline Shuttle running 3x10 meters -30 meters running, seconds & $4,15 \pm 0,09$ & 49,05 \\
\hline Zigzag running -30 meters running & $2,93 \pm 0,15$ & \multicolumn{2}{|c|}{$4,00 \pm 0,11$} \\
\hline
\end{tabular}

Football players from the EG overcame 30 meters running with straight dribbling. They had valid advantages over football players from the CG in time of dribbling with shuttle running and zigzag running (table 2).

Table 2. Quality indices of mastering the technique of dribbling and stroke after the pedagogical experiment, $\mathrm{X} \pm \delta$

\begin{tabular}{|l|c|c|c|}
\hline \multicolumn{1}{|c|}{ Testing exercises } & \multicolumn{2}{|c|}{ Groups of respondents } & \multirow{2}{*}{$\mathrm{P}$} \\
\cline { 2 - 3 } & $\mathrm{CG}, \mathrm{n}=18$ & $6,68 \pm 0,30$ & $\leq 0,05$ \\
\hline Dribbling 30 meters, seconds & $6,91 \pm 0,33$ & $11,05 \pm 1,54$ & $\leq 0,05$ \\
\hline Dribbling in shuttle running 3x10 meters, seconds & $12,23 \pm 1,89$ & $10,33 \pm 1,02$ & $\leq 0,05$ \\
\hline Dribbling in zigzag running, seconds & $11,40 \pm 1,57$ & $1,03 \pm 0,11$ & $\leq 0,05$ \\
\hline $\begin{array}{l}\text { difference (time of dribbling 30 meters - time of running 30 meters), } \\
\text { seconds }\end{array}$ & $1,21 \pm 0,10$ & $1,40 \pm 0,15$ & $\leq 0,05$ \\
\hline $\begin{array}{l}\text { difference (time of dribbling in shuttle running 3x10 meters - time of } \\
\text { shuttle running 3x10 meters), seconds }\end{array}$ & $2,38 \pm 0,20$ & $1,93 \pm 0,22$ & $\leq 0,05$ \\
\hline $\begin{array}{l}\text { Difference (time of zigzag dribbling- time of zigzag running), sec- } \\
\text { onds }\end{array}$ & $2,77 \pm 0,24$ & 12 of 16 (75\%) & $\leq 0,05$ \\
\hline Stroke, amount / \% of successful attempts & 9 of 18 (50\%) \\
\hline
\end{tabular}

The results of 30 meters running with dribbling increased the results in 30 meters running for 1,03 s, at the same time, in football players from the CG this index was validly higher - 1,21 s. It proves that football players from the EG after the experiment mastered the technique of straight dribbling better than football players from the CG. Low indices of the difference between the results of straight dribbling at 30 meters distance and the time of dribbling in shuttle running (1,40 against 2,38 seconds among football players from the $\mathrm{CG}$ ) and the time of dribbling in zigzag running (1,93 against 2,77 seconds) prove the fact that football players from the EG had mastered the technique of dribbling with speed and direction change better. Football players from the EG coped with testing exercises of "stroke" better: from 16 people $12(75 \%)$ were successful fulfilling this test exercise.

We can explain presented above results of the pedagogical experiment. ITC considerably broadens the opportunities of methodical techniques of repeated, adjacent, closed up, contrast and variative tasks use. Its use helps not only to give the route of moving, but also the distance of pushing the ball during each successive touch. This distance can be the same, contract, gradually closed up, close or variatively changing. Fulfilling dribbling in terms of these methodical techniques, a football player develops the ability to differentiate muscle efforts depending on the distance, for which it is necessary to push the ball, develops the ability to influence the ball with the effort, when each gradual touch wouldn't lead to considerable speed of running and the length of the running step change.

Another advantage of the innovative methodology is the ability to realize an operational control over the quality of the training tasks fulfillment. The moving characteristic of a football player and a ball are registered with the help of video cameras and are transferred for saving and analysis in computer with further display. The characteristics of the activity correspondence with the set parameters of the training tasks is transferred to a football player with the help of ITC giving light or sound signals.

ITC use helps to regulate the difficulty if the training tasks fulfillment in accordance with individual differences in mastering techniques of dribbling and stroke. The regulated parameters of the training tasks are the created on the playing ground characteristics of light objects: their amount, volume, speed of movement, degree of ambiguity of speed and direction of movements change, the degree of their inertia.

Typical for ITC abilities of registering objective quality indices of the training tasks fulfillment create the conditions for their fulfillment in terms of competitive method.

\section{Conclusions}

Created by us ITC provides the following: creation with the help of technical means dynamically changing net and sound design of the training sphere, which sets spatio-temporal conditions of an athlete's movements with the ball and without it and in terms of opposition with the opponent; the parameters of a functional state registration and the training task fulfillment. They are fixed in specially organized database. Operative control over the training sphere formation is realized manually (by a coach), according to before set or formed program. It takes into account the results of athlete's readiness indices, functional state and activity diagnostics. The training sphere design includes contrastly defining dynamically lit objects: spots, lines, circles, corridors, ladders, chess squares; sound signals for loud-speaking communication with a coach provision or for background music presentation. 
ITC use helps to broaden the range of training means for the skills of movement formation without the ball with unexpected speed and direction change, for the ability development to differentiate muscle efforts while influencing the ball, measuring them with the length and speed of the running step; for the skills of dribbling formation with speed and direction of movement change, the skills of stroke formation. Owing to ITC use the following conditions appear: for methodical techniques of repeated, adjacent, closed up, contrast and variative tasks use; an operative control realization over the quality of the training tasks fulfillment, the difficulty of the training tasks fulfillment regulation, taking into account individual educational abilities of football players; for the training tasks fulfillment in terms of competitive method.

\section{References}

1. National strategy of football development in the Russian Federation during the period till 2030, URL: https://rfs.ru/projects/rfs/razvitie-futbola-v-rf-2030/documents

2. G.L. Drandrov, N.Kh. Kudyashev, Developing the methodology of technical training of young football players (Chuvash State Pedagogical University, Cheboksary, 2011)

3. V.K. Balsevich, Theory and practice of physical culture, 4, 21-40 (1999)

4. V.K. Balsevich, Theory and practice of physical culture, 10, 5-6 (2000)

5. A.L. Dimova, Theory and practice of physical culture and sport, 12, 40-43 (2011)

6. V.A. Danilchenko, T.A. Khabinets, Yu.L. Khlevna, Physical upbringing of students, 3, 29-32 (2012)

7. O.I. Pavlova, M.G. Faskhutdinov, M.V. Buvalin, Theory and practice of physical culture, 11, 86-87 (2010)

8. A.A. Suchilin, I.N. Novokshchenov, The Russian Journal of Physical Education and Sport, 13(1), 46-52 (2018). DO I 10/14526/012018282

9. V.E. Afonshin, The Eurasian Patent Organization 201300603/26, WO2012096594 A1, Method for training players and sportsmen

10. V.E. Afonshin, United States Patent Application Publication US 2013/0288213 A1. Method for training players and sportsmen 